\title{
Integral equation theory approach to rodlike polyelectrolytes: Counterion condensation
}

\author{
T. Hofmann a) \\ Abteilung Theoretische Physik, Universität Ulm, D-89069 Ulm, Germany \\ R. G. Winkler \\ Institut für Festkörperforschung, Forschungszentrum Jülich, D-52425 Jülich, Germany \\ P. Reineker \\ Abteilung Theoretische Physik, Universität Ulm, D-89069 Ulm, Germany
}

(Received 13 December 2000; accepted 15 March 2001)

\begin{abstract}
We investigate the structural properties of rigid linear polyelectrolytes in dilute and semidilute solutions using an integral equation theory. The Polymer Reference Interaction Site Model together with the Reference Laria Wu Chandler Closure is solved numerically taking the counterions into account explicitly. The counterions and the polymer chains, modeled as linearly connected, charged hard spheres, interact through an unscreened Coulomb potential. The pair correlation functions between the monomers of different chains, the counterions, and the monomers and counterions, respectively, are calculated for various densities and Bjerrum lengths. Based upon these quantities, the effective potential among the monomers and the counterions, respectively, is extracted. In particular, a critical Bjerrum length is determined, which separates the regime of a repulsive interaction between the counterions from the regime of an attractive interaction transmitted by the polymer chains. (C) 2001 American Institute of Physics. [DOI: 10.1063/1.1370075]
\end{abstract}

\section{INTRODUCTION}

The study of polyelectrolytes, i.e., charged polymers dissolved in a polar solvent and in the presence of dissociated counterions, has been an outstanding problem in polymer science for several decades, both from an experimental as well as a theoretical point of view. ${ }^{1-5}$ The importance of this class of polymers is quite obvious considering that the two perhaps best known biopolymers, namely DNA and RNA, are polyelectrolytes. The technical application of synthetic polyelectrolytes, such as sulfonated polystyrene or polyacrylic acid, are very widespread, ranging from water purification through extraction of ions to stabilization of gels and micelles and even to the use as an absorbent material for diapers. ${ }^{1-3,6}$ Despite the theoretical and experimental efforts many properties of polyelectrolytes are, in comparison to neutral polymers, still poorly understood. ${ }^{7-12}$ Experiments on polyelectrolyte solutions using different methods often lead to controversial results. ${ }^{7}$ Furthermore, experimental results on single polyelectrolyte chains are still lacking due to the immense problems caused by trace impurities and very low scattering intensities when measuring dilute solutions. ${ }^{3,13}$ Therefore, scattering experiments and measurements of the radius of gyration are almost always performed in the semidilute regime. ${ }^{14-18}$

From the theoretical point of view the main problem in understanding polyelectrolyte solutions is the long range Coulomb interaction. Renormalization group theories and scaling ideas, which have proved to be very successful for neutral polymer solutions, ${ }^{19-21}$ are now difficult to apply. The long range Coulomb interaction, together with screening

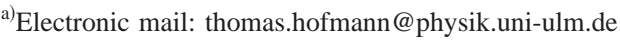

effects caused by the counterions, introduces more than one new length scale in the problem, which means that short range (hard core) and medium to long range interactions are simultaneously present. This coupling of different length scales leads to a severe influence of the local chain properties on the properties of the whole system. ${ }^{22-24}$

Computer simulations of polyelectrolyte solutions are very time consuming, since the adequate treatment of the long range Coulomb potential requires techniques like the Ewald summation. ${ }^{25-28}$ Therefore, computer simulations are often limited to short chains and/or dilute solutions or even single chains. ${ }^{29-31}$ Nevertheless, computer simulations can give deeper insight to polyelectrolyte systems, e.g., the counterion distribution or chain conformations. ${ }^{32}$

Another theoretical approach to polyelectrolyte solutions is the Polymer Reference Interaction Site Model (PRISM) theory. The PRISM theory, which has been very successfully applied to neutral polymers, ${ }^{33-39}$ was first introduced by Curro and Schweizer. ${ }^{35}$ The first applications of the PRISM theory to polyelectrolyte solutions were based on a very simple one-component model, which treated the counterions within the Debye-Hückel approximation. ${ }^{40-42}$ This simple model proved to be very useful to obtain the thermodynamic and structural properties. In particular, the scaling behavior of the first peak in the polymer structure factor could be explained by that approach for a wide range of parameters such as chain length, density, and Bjerrum length. However, this model is lacking some major features required for a complete understanding of polyelectrolyte solutions. In the one-component model the distribution of the counterions is always implicitly assumed to be spherically symmetric. This approximation may be valid for weakly charged systems, but is clearly wrong for strong Coulomb interactions, when 
counterion condensation is relevant. In the latter case some or even all counterions are bound to the chain due to the strong electrostatic coupling between the monomers and counterions and only those counterions not condensed on the chain may be described in the Debye-Hückel approximation. Hence, counterion condensation cannot be described in the one-component model. The growing interest in recent years in the distribution of the counterions around the chain and especially counterion condensation requires a more sophisticated model. Such a model is the so called primitive model. ${ }^{43}$ Here the counterions are taken into account explicitly and all ionic species, i.e., the monomers and counterions, interact via hard core repulsion and an unscreened Coulomb potential. Recent studies of polyelectrolyte systems ${ }^{44-47}$ based on this model have mainly concentrated on the variation of the density of the solution.

Various criteria are used to characterize counterion condensation. Since the counterions are condensed on a polymer chain a measure of the amount of condensed counterions is obtained by counting their number within a certain distance around a polymer (or monomer).

Alternatively, the electrostatic binding energy might be used to determine a distance within which the ions are declared to be condensed. Both of these methods have their shortcomings as discussed in Ref. 48 for a rod-like polymer. Instead, in Refs. 48 and 49 an inflection point criterion is suggested to define the critical Bjerrum length and the amount of condensed ions. However, to apply this idea to a dilute or semidilute systems is rather difficult, since the criterion requires the association of a particular ion with a particular polymer (at least partially). It is this step which causes major difficulties in a three dimensional system. ${ }^{44}$ Hence, it is not obvious yet, whether the criterion is useful at all.

In this paper, we address the structural properties of polyelectrolyte solutions for various Bjerrum lengths, chain lengths, and densities. In particular, we demonstrate that the counterion-counterion pair correlation function can be used to define a critical Bjerrum length, which separates the regime of a repulsive interaction between the counterions and an attractive regime transmitted via the polymer chains.

The paper is organized as follows. In Sec. II the chain model and the basic features of the PRISM theory are described. In Sec. III results for the correlation functions are presented. In Sec. IV we calculate the effective potential between counterions and between monomers, respectively. In Sec. V we focus on the counterion condensation and present the criteria for the critical Bjerrum length above which counterion condensation is expected. Finally, Sec. VI summarizes our results.

\section{PRISM AND MODEL}

\section{A. PRISM}

The PRISM theory is a liquid state theory based on modified Ornstein-Zernike integral equations. ${ }^{50}$ By taking the connectivity of the monomers of a chain molecule into account, the RISM theory, first proposed by Chandler and Andersen ${ }^{51}$ for treating solutions of diatomic molecules, was extended to the PRISM theory by Curro and Schweizer. ${ }^{35}$
The PRISM theory as well as the Ornstein-Zernike equation connects the total correlation function $h(r)$, which is related to the well known pair correlation function $g(r)$ via $h=g$ -1 , with the direct correlation function $c(r)$ and, in the case of polymers with intramolecular correlation functions, the intramolecular structure factor. For the primitive model studied in this paper three different correlation functions are relevant: the monomer-monomer, the counterion-counterion, and the monomer-counterion correlation function. The numerical treatment of the PRISM equations faces, especially for long polymer chains, a severe problem. In the original formulation of the PRISM equation all monomers of the chain are treated explicitly, which means that a vast number of coupled equations has to be solved. This problem can be solved if we neglect chain end effects and hence consider all monomers on the chain as equivalent. In this approximation the $N^{2}$ intermolecular correlation functions $g_{\mathrm{mm}}^{i j}$, where $N$ is the number of monomers of a chain and $i, j$ are the indices of the individual monomers on different chains, reduce to a single intermolecular correlation function $g_{\mathrm{mm}}$ between monomers. Thus, the PRISM equations can conveniently be written in Fourier space as

$$
\begin{aligned}
h_{\mathrm{mm}}(k) & =\frac{\omega(k)}{\Delta(k) \rho_{m}}\left(1-\rho_{c} c_{\mathrm{cc}}(k)-\Delta(k)\right), \\
h_{\mathrm{cc}}(k)= & \frac{1}{\Delta(k) \rho_{c}}\left(1-\rho_{m} \omega(k) c_{\mathrm{mm}}(k)-\Delta(k)\right), \\
h_{\mathrm{mc}}(k)= & \frac{\omega(k)}{\Delta(k)} c_{\mathrm{mc}}(k), \\
\Delta(k)= & 1-\rho_{c} c_{c c}(k)-\rho_{m} \omega(k) c_{\mathrm{mm}}(k)+\rho_{m} \rho_{c} \omega_{m}(k) \\
& \times\left(c_{\mathrm{mm}}(k) c_{\mathrm{cc}}(k)-c_{\mathrm{mc}}^{2}(k)\right),
\end{aligned}
$$

where $\rho_{m}$ and $\rho_{c}$ are the monomer and counterion densities, respectively, and $\omega(k)$ denotes the single chain intramolecular structure factor. For symmetry reasons the monomercounterion $\left(g_{\mathrm{mc}}\right)$ and counterion-monomer $\left(g_{\mathrm{cm}}\right)$ correlation functions are equivalent. The chain model itself enters solely through the intramolecular structure factor. It can easily be calculated for a fixed chain conformation or has to be determined in a self-consistent manner if conformational changes of a chain have to be taken into account. Once the correlation functions are known, the structure factors of the solution can be calculated in a straightforward manner:

$$
\begin{aligned}
& S_{\mathrm{mm}}(k)=\omega(k)+\rho_{m} h_{\mathrm{mm}}(k), \\
& S_{\mathrm{cc}}(k)=1+\rho_{c} h_{\mathrm{cc}}(k), \\
& S_{\mathrm{mc}}(k)=\rho_{m} h_{\mathrm{mc}}(k) .
\end{aligned}
$$

The PRISM equations can be solved, if additional equations are available which connect the correlation functions with the intermolecular pair potentials. These so called closure relations are given exactly for hard core systems by $g(r)$ $=0$ for $r<\sigma$, where $\sigma$ is the diameter of the hard sphere. Unfortunately, no exact closure relation exists for $r>\sigma$ and approximations are required. Many such closures have been proposed in the literature during the last decades. ${ }^{37,50}$ They can be divided roughly into two groups: The atomic closures, 
such as Percus-Yevick (PY), hypernetted chain, and mean spherical approximation are based on the theory of simple, atomic liquids, ${ }^{52-54}$ whereas the molecular closures, such as reference molecular hypernetted chain, Laria Wu Chandler (LWC), or reference Laria Wu Chandler (RLWC), take the connectivity of the monomers into account. ${ }^{37,55}$ It has been shown that the RLWC closure is a valid closure for polyelectrolyte systems. ${ }^{42}$ The RLWC closure is given by

$$
\begin{aligned}
\omega_{i}(r) * & c_{i j}(r) * \omega_{j}(r) \\
= & \omega_{i}(r) * c_{o, i j}(r) * \omega_{j}(r)-\omega_{i}(r) * \beta v_{i j}(r) * \omega_{j}(r)+h_{i j}(r) \\
& -h_{o, i j}(r)-\ln \left(\frac{g_{i j}(r)}{g_{o, i j}(r)}\right), i, j \in\{m, c\},
\end{aligned}
$$

where the index 0 denotes reference functions obtained for a pure hard core system for the same densities with the PY closure and the asterisks denotes convolution integrals. It should be noted that in our notation $\omega_{m}(k)=\omega(k)$ and $\omega_{c}(k)=1$. The set of coupled integral equations together with the appropriate closures is solved iteratively until convergence is achieved using a Picard iteration scheme. ${ }^{50}$ The convergence is usually quite fast and requires only a few minutes for a given set of parameters on a standard personal computer.

\section{B. Model of the system}

The polyelectrolyte chains are modeled as a collection of $N$ charged hard spheres with diameter $\sigma_{m}$ separated by a fixed distance $l$ and charge $Z_{m} e$. Since we consider rodlike chains, the spheres are arranged in a linear configuration. For this model no conformational changes have to be considered as the chain remains in its rigid rod configuration for all parameter variations. Hence, the intramolecular structure factor is given by

$$
\omega(k)=1+\frac{2}{N} \sum_{j=1}^{N-1}(N-j) \frac{\sin (j k l)}{j k l} .
$$

The counterions are also modeled as charged hard spheres with diameter $\sigma_{c}$ and a charge of $Z_{c} e$. Charge neutrality requires that the monomer density $\rho_{m}$ and the counterion density $\rho_{c}$ fulfill the equation $Z_{m} \rho_{m}+Z_{c} \rho_{c}=0$. The influence of the solvent is treated in a mean field manner. It is described as a homogeneous dielectric continuum with the dielectric constant $\epsilon$. The pair interaction potential for all ionic species is given by

$$
\beta v_{i j}(r)=\beta v_{i j}^{\mathrm{HC}}(r)+Z_{i} Z_{j} \frac{l_{B}}{r} ; i, j \in\{m, c\},
$$

where $v_{i j}^{\mathrm{HC}}(r)$ is the hard core potential and $l_{B}=\beta e^{2} / \epsilon$ is the Bjerrum length.

\section{CORRELATION FUNCTIONS}

The following results were obtained for systems with monovalent counterions, i.e., $Z_{c}=-1$ and single charged monomers $Z_{m}=+1$. In this case charge neutrality demands $\rho_{m}=\rho_{c}$, therefore we use in the following $\rho=\rho_{c}$. Furthermore we set $\sigma_{m}=\sigma_{c}=\sigma$, i.e., the counterions are of the
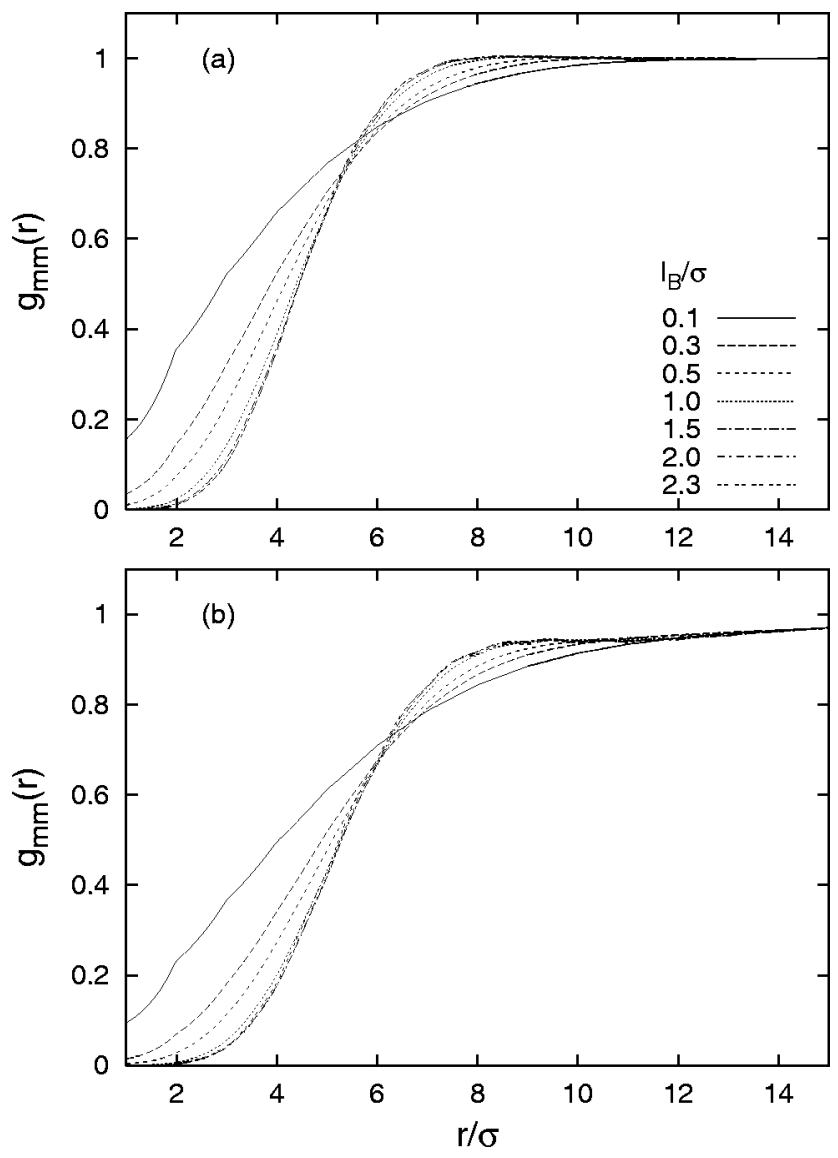

FIG. 1. Monomer-monomer pair correlation function $g_{\mathrm{mm}}(r)$ for various Bjerrum lengths $l_{B}$. The density is $\eta=10^{-2}$ and the chain lengths are $N$ $=10$ (a) and $N=80(\mathrm{~b})$.

same size as the monomers. Moreover, in our calculations the segment length $l$ is equal to the hard core diamater $\sigma$. Instead of the density $\rho$ we will often use the dimensionless packing fraction $\eta=\pi \rho \sigma^{3} / 6$. Unfortunately, the numerical iteration scheme required for solving the PRISM equations does not always converge. This is especially true for highly charged systems and depends on density. The usage of other closures leads to the same problems even at smaller Bjerrum lengths. Hence the limitation of the parameter range seems to be a consequence of the closure and not of the numerical iteration scheme. As already mentioned above, the density dependence of the relevant properties has already been discussed in detail in other publications. ${ }^{44-46}$ Therefore, we will mainly discuss the influence of the Bjerrum and chain lengths on the structure of the system.

\section{A. Monomer-monomer correlation}

Figure 1 presents the monomer-monomer pair correlation function for various Bjerrum lengths ranging from very weakly charged systems up to well above the Manning threshold for counterion condensation $\left(l_{B} / \sigma=1\right)$. The packing fraction is $\eta=10^{-2}$. Results for chain lengths $N=10$ (a) and $N=80$ (b) are presented. In contrast to the variation of the density, which has a rather large influence on the structure and therefore on the monomer-monomer correlation function, the variation of the Bjerrum length causes only minor changes in $g_{\mathrm{mm}}$. Figure 1 shows that the contact value 

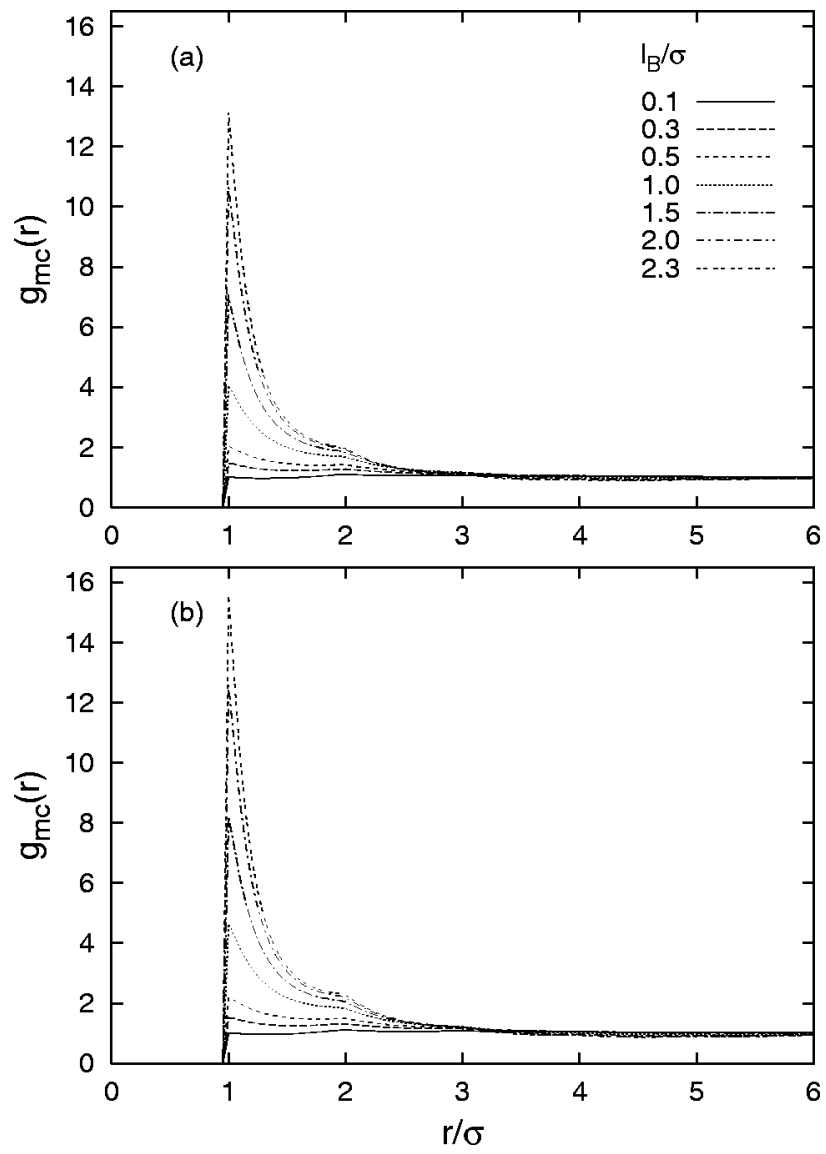

FIG. 2. Monomer-counterion pair correlation function $g_{\mathrm{mc}}(r)$ for various Bjerrum lengths $l_{B}$. The density is $\eta=10^{-2}$ and the chain lengths are $N$ $=10$ (a) and $N=80(\mathrm{~b})$.

$g_{\mathrm{mm}}(\sigma)$ decreases with increasing $l_{B}$ and simultaneously a small peak arises at $r \approx 7 \sigma$. Notice that all curves for the different values of $l_{B}$ cross each other at the same distance $r$. A detailed examination shows that this distance increases with decreasing density. A similar calculation within the Debye-Hückel approximation yields almost indistinguishable results, which suggests that increasing $l_{B}$ not only increases the bare repulsion among the monomers but also induces a screening by the counterions. Furthermore, Fig. 1 shows that the monomer-monomer pair correlation functions of the two different chain lengths are virtually indistinguishable. This has to be expected as the density is on the order of the overlap concentration given by $\rho^{*}=1 / N^{2}$.

Our calculations confirm the scaling relations of the density dependence of the position $k_{\max }$ of the first peak in the monomer-monomer structure factor. ${ }^{56-59}$ However, inbetween the two known regimes we find an additional large density range with a scaling exponent different from the known ones, particularly for long chains. A more detailed discussion of this issue will be presented elsewhere.

\section{B. Monomer-counterion correlation}

The distribution of the counterions around the monomers of a chain can be described by the monomer-counterion correlation function $g_{\mathrm{mc}}$. Figure 2 displays $g_{\mathrm{mc}}$ for different Bjerrum lengths $l_{B}$ at the density $\eta=10^{-2}$ and the chain
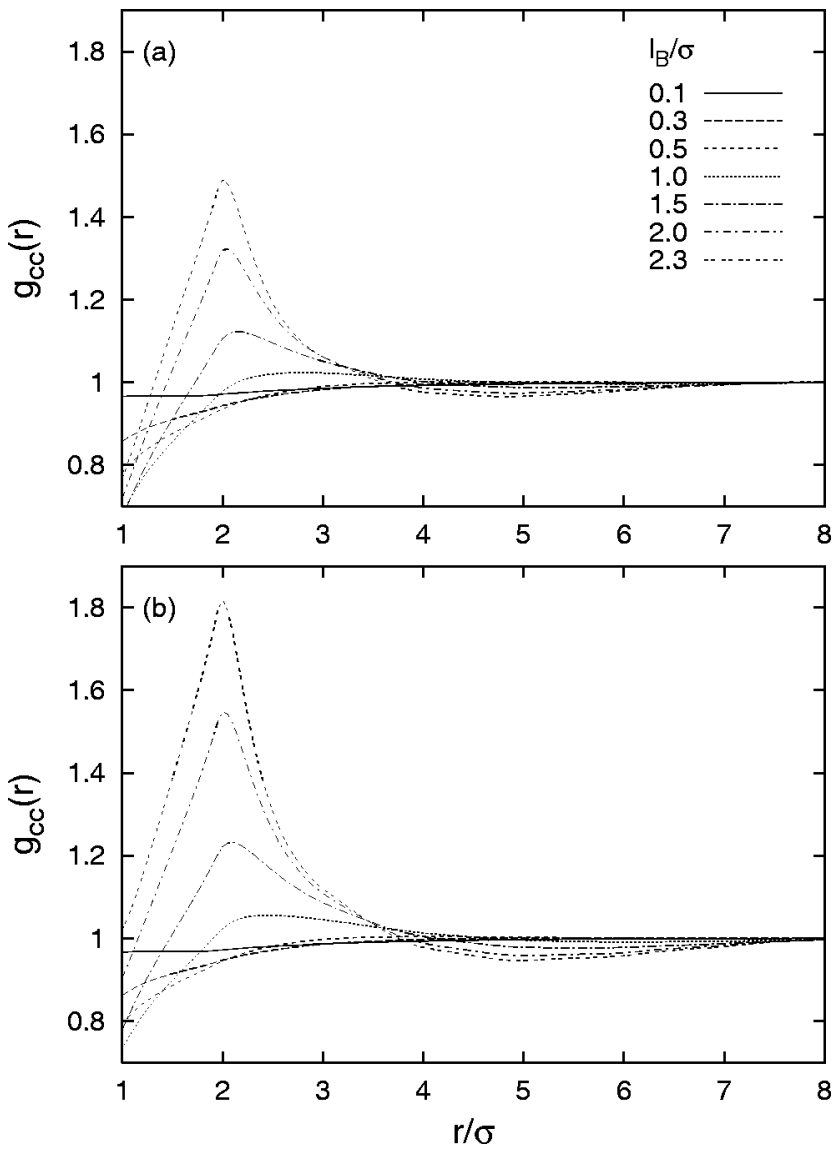

FIG. 3. Counterion-counterion pair correlation function $g_{\mathrm{cc}}(r)$ for various Bjerrum lengths $l_{B}$. The density is $\eta=10^{-2}$ and the chain lengths are $N$ $=10(\mathrm{a})$ and $N=80(\mathrm{~b})$.

lengths $N=10$ (a) and $N=80$ (b). For small $l_{B} g_{\mathrm{mc}}(r)$ is nearly constant with a value of about one for all $r$. Hence, the local counterion density matches the bulk density almost everywhere, i.e., the counterions are distributed homogeneously over the whole system. With increasing Bjerrum length an increasing peak appears at $r=\sigma$ and a much smaller peak at $r=2 \sigma$. This implies that the counterions are now no longer homogeneously distributed, but are found with larger probability in the vicinity of the chains. It should be noted that the distance $r=\sigma$ is the smallest possible distance between monomers and counterions because of the hard core repulsion. The modulations of $g_{\mathrm{mc}}$ clearly reflect counterion condensation (see discussion in Sec. IV). An interesting fact can be observed by comparing Figs. 2(a) and 2(b) for the two values of the chain lengths. The height of the peak in $g_{\mathrm{mc}}$ increases with increasing monomer number $N$. Since the pair correlation function is directly connected to the number of counterions per monomer it is obvious from this figure that for longer chains more counterions per monomer are condensed than for shorter chains at the same Bjerrum length.

\section{Counterion-counterion correlation}

The counterion-counterion correlation function $g_{\text {cc }}$ provides a deeper insight into the issue of counterion condensation. Figure 3 displays $g_{\text {cc }}$ for different Bjerrum lengths and 
two chain lengths, $N=10$ (a) and $N=80$ (b), at the density $\eta=10^{-2}$. For very low $l_{B} g_{\text {cc }}$ is constant for almost all distances, corresponding to a homogeneous distribution of the counterions. An increase of the Bjerrum length first leads to a decrease of the contact value $g_{\mathrm{cc}}(\sigma)$ and the overall function value for short distances. A further increase of $l_{B}$ reverts this behavior and a peak appears at $r=2 \sigma$. The height of the peak increases with increasing $l_{B}$ and its width decreases. This behavior can be explained by counterion condensation. For $l_{B}$ well below the critical Bjerrum length for counterion condensation, an increase of $l_{B}$ results in stronger repulsion of the counterions. Therefore the counterions are repelled from each other corresponding to a decrease of $g_{\mathrm{cc}}$ at small length scales. If the Bjerrum length is further increased the counterions are attracted by the polyion and start to condensate on the chain, which must be accompanied by a decrease of the mean separation between ions. Consequently, $g_{\mathrm{cc}}$ starts to increase at short distances. Hence, despite the repulsive Coulomb interaction the counterions are, for sufficiently strong interactions, subject to an effective attractive potential next to a polymer chain. This aspect is discussed in detail in Sec. IV. The position of the peak at $r=2 \sigma$ is quite simple to explain if we consider the configuration with the lowest energy. The electrostatic interaction forces the condensed ions to be as close to the monomers as possible but at the same time as far apart as possible from each other. This is achieved, if the counterions are located on opposite sites of the chain monomers. From Fig. 3 follows that the Bjerrum length, which corresponds to the turning point in the behavior of $g_{\text {cc }}$ described above, is lower for longer chains. This is in agreement with the fact discussed previously that longer chains carry more condensed ions per monomer than shorter chains at the same Bjerrum length.

\section{EFFECTIVE POTENTIAL}

The behavior of the counterion-counterion correlation function, as discussed in Sec. IIIC, suggests that the effective potential between the counterions is attractive above a certain Bjerrum length due to counterion condensation. The effective potential between two counterions themselves can be calculated if the multicomponent model with polymers and counterions is reduced to a simpler model consisting only of counterions. We define this simple model in such a way that the effective potential between the counterions of the new system yields exactly the same correlation function $g_{\mathrm{cc}}$ as found in the multicomponent case at the same (counterion) density. Compared to a multicomponent system, where the correlation functions are calculated from a known potential, we now calculate the potential from a known correlation function. Starting from the counterion-counterion correlation function $g_{\text {cc }}$ of the multicomponent model we calculate an effective direct correlation function $c_{\text {eff }}$ via the one-component Ornstein-Zernike equation:

$$
c_{\mathrm{cc}, \mathrm{eff}}(k)=\frac{h_{\mathrm{cc}}(k)}{\omega_{c}^{2}(k)+\rho \omega_{c}(k) h_{\mathrm{cc}}(k)} .
$$

The RLWC closure for the one-component model of counterions is given by

$$
\begin{aligned}
\omega_{c}(r) * c_{\mathrm{cc}, \mathrm{eff}}(r) * \omega_{c}(r) & \\
= & \omega_{c}(r)^{*} c_{o, \mathrm{cc}, \mathrm{eff}}(r) * \omega_{c}(r)-\omega_{c}(r) * \beta v_{\mathrm{cc}, \mathrm{eff}}(r) * \omega_{c}(r) \\
& +h_{\mathrm{cc}}(r)-h_{o, \mathrm{cc}, \mathrm{eff}}(r)-\ln \left(\frac{g_{\mathrm{cc}}(r)}{g_{o, \mathrm{cc}, \mathrm{eff}}(r)}\right),
\end{aligned}
$$

where $c_{o, \text { cc,eff }}$ and $g_{o, \text { cc,eff }}$ denote reference functions obtained for a pure one-component hard core system with the PY closure at the same density. It should be noted that the correlation functions $h_{\mathrm{cc}}$ and $g_{\mathrm{cc}}$ are, by definition of the effective potential, the same as the correlation functions of the multicomponent model. Subtracting this equation from the RLWC closure of the multicomponent system and extracting the effective potential, we obtain

$$
\begin{aligned}
\beta v_{\mathrm{cc}, \mathrm{eff}}(r)=\beta v_{\mathrm{cc}}(r)+ & \left(c_{\mathrm{cc}}(r)-c_{\mathrm{cc}, \mathrm{eff}}(r)\right)-\left(c_{o, \mathrm{cc}}(r)\right. \\
\left.-c_{o, \mathrm{cc}, \mathrm{eff}}(r)\right)-F_{\mathrm{cc}}(r), & \\
\omega_{c}(r) * F_{\mathrm{cc}}(r) * \omega_{c}(r)= & \left(h_{o, \mathrm{cc}, \mathrm{eff}}(r)-h_{o, \mathrm{cc}}(r)\right) \\
+ & \ln \left(\frac{g_{o, \mathrm{cc}}(r)}{g_{o, \mathrm{cc}, \mathrm{eff}}(r)}\right)
\end{aligned}
$$

For low and moderate densities (smaller than the overlap density) the reference functions of the multicomponent and the one-component model are almost equal and hence the effective potential is in good approximation given by

$$
\beta v_{\text {cc,eff }}(r)=\beta v_{\text {cc }}(r)+\left(c_{\mathrm{cc}}(r)-c_{\text {cc,eff }}(r)\right) \text {. }
$$

Therefore, the effective potential is equal to the bare potential plus a modification given by the difference in the direct correlation functions of the multicomponent and onecomponent model. Figure 4 shows the effective counterioncounterion potential obtained in the way described above for different Bjerrum lengths and two chain lengths, $N=10$ (a) and $N=80$ (b), at the density $\eta=10^{-2}$. As is obvious from this figure, the effective potential is purely repulsive for low values of $l_{B}$ and can very well be approximated by the bare Coulomb potential between the counterions. As the Bjerrum length increases the potential becomes negative for distances larger than a certain critical distance, leading to an attractive force between two counterions. For even larger values of $l_{B}$, the effective potential exhibits a distinct minimum at a distance of about $r=2 \sigma$ in agreement with the position of the peak in $g_{\mathrm{cc}}$. Moreover, Fig. 4 shows that the minimum of the potential at $r=2 \sigma$ is deeper for longer chains, i.e., the attractive force between two counterions is stronger in a system with longer polymer chains than in a system with shorter ones. The transition from a repulsive to an attractive effective potential may also be used to define a critical Bjerrum length for counterion condensation. Investigation along this line are underway.

The method described above also allows us to calculate the effective potential among the monomers. The equation for the effective potential is identical to the one of the effective counterion potential, if we just replace the index $c$ by $m$. Figure 5 displays the effective monomer-monomer potential calculated in the way described above at the density $\eta$ $=10^{-2}$ and for various Bjerrum lengths. The chain lengths are $N=10$ (a) and $N=80$ (b). Similar to the counterioncounterion potential, we observe a minimum at $r \approx 2 \sigma$ for Bjerrum lengths $l_{B}>1.5 \sigma$. Hence, the monomers attract each 

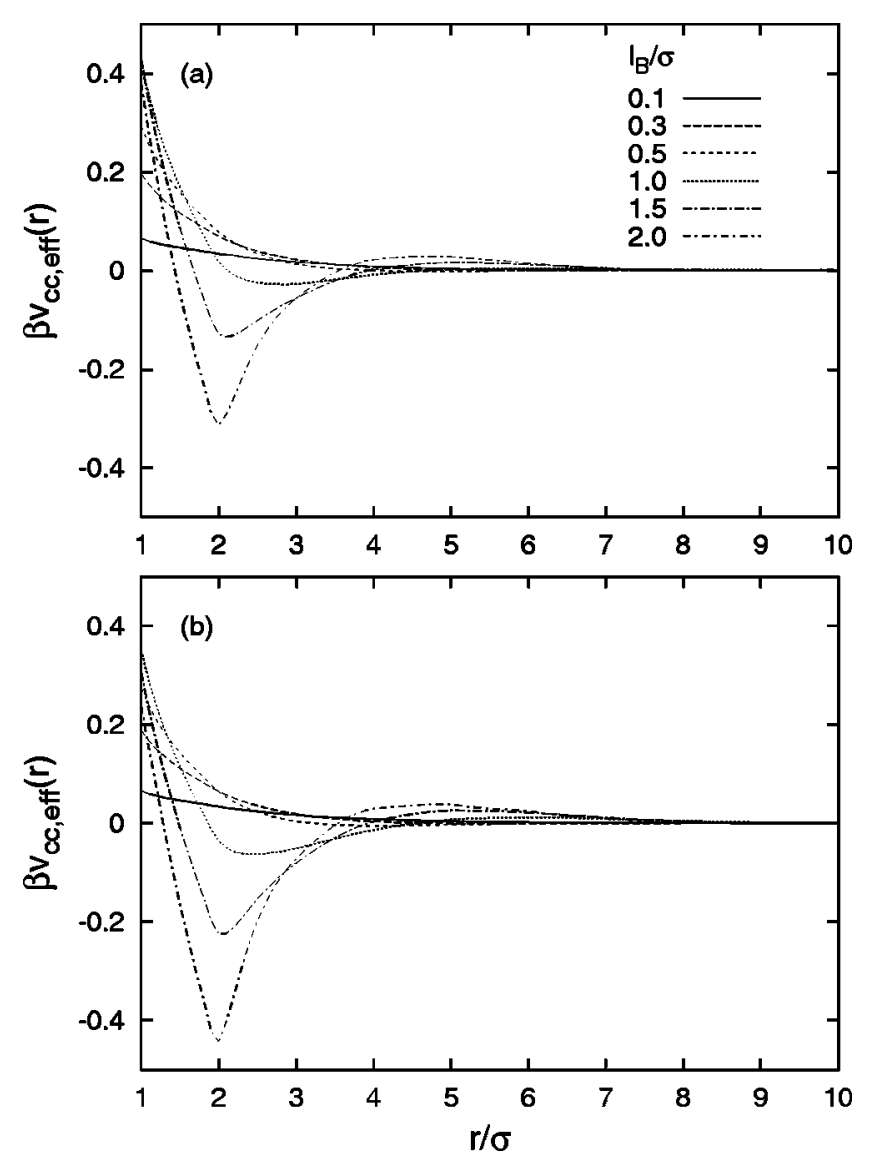

FIG. 4. Effective potential between two counterions for various Bjerrum lengths $l_{B}$. The density is $\eta=10^{-2}$ and the chain lengths are $N=10$ (a) and $N=80$ (b).

other at high interaction strengths and small distances. A similar behavior has been reported for multivalent ions. ${ }^{60}$ Figure 6 shows that for low and moderate Bjerrum lengths the effective potential is a monotonous decreasing function for $r>2 \sigma$ in agreement with the Debye-Hückel approach. For $r<2 \sigma$ we find deviations from the Debye-Hückel potential due to the depletion interaction. ${ }^{61}$ At high Bjerrum lengths $\left(l_{B}>\sigma\right)$ the effective potential still decays in the average as predicted by the Debye-Hückel model. Apart from the attractive interaction among the monomers $(r \approx 2 \sigma)$, however, we observe pronounced modulations on the length scale of the diameter of a monomer. These modulations are also caused by the depletion interaction. The quantitative comparison between our calculations and the Debye-Hückel potential exhibits excellent agreement for the screening length as well as the dependence of that potential on the interaction strength. The deviations from the Debey-Hückel representation of the interaction among the monomers is not surprising. The condensation of the counterions leads to a screening of the Coulomb interaction which is not captured by the Debey-Hückel potential.

\section{COUNTERION CONDENSATION}

From the discussions above the question arises for which Bjerrum lengths counterion condensation can be expected. As we already pointed out there exists a Bjerrum length
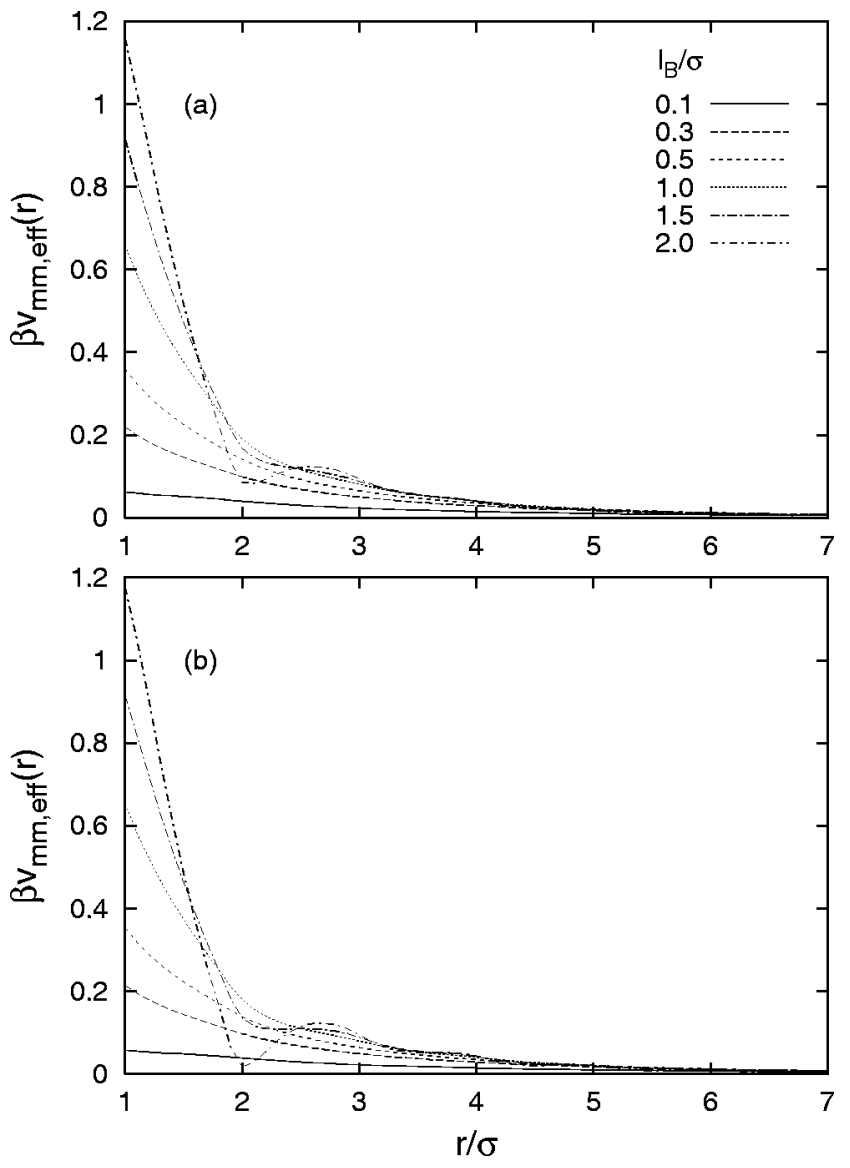

FIG. 5. Effective potential between two monomers for various Bjerrum lengths $l_{B}$. The density is $\eta=10^{-2}$ and the chain lengths are $N=10$ (a) and $N=80($ b).

separating two different regimes in the behavior of $g_{\mathrm{cc}}$. For $l_{B}$ below this value the counterions simply repel each other because of the Coulomb interaction. Above this value, however, the effective potential between the counterions is attractive and the ions condense on the chain. Therefore the average number of counterions within a given distance $\hat{r}$ of a counterion is first decreasing with increasing $l_{B}$ and for $l_{B}$

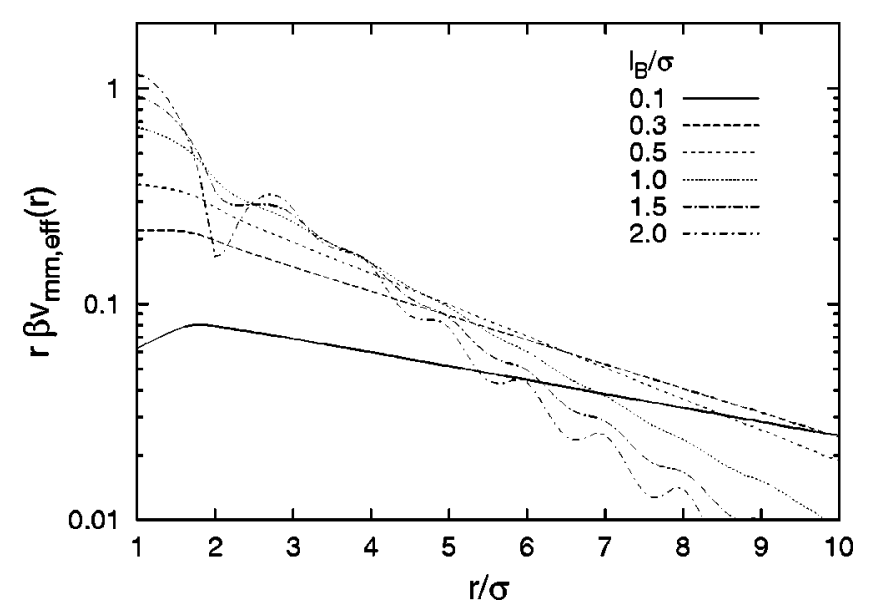

FIG. 6. Scaled effective potential $\left(\log r v_{\mathrm{mm}, \text { eff }}\right)$ between two monomers for various Bjerrum lengths $l_{B}$ at the density $\eta=10^{-2}$. The chain length is $N$ $=80$. 


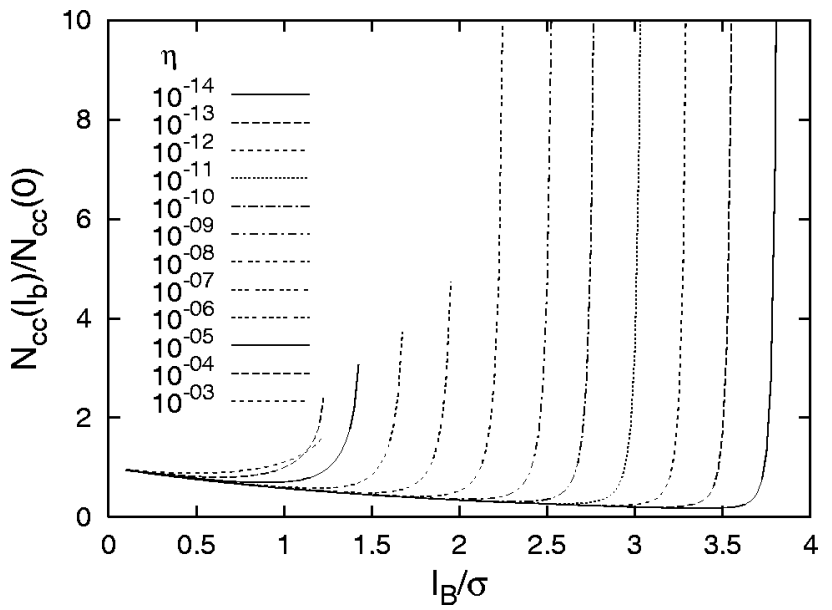

FIG. 7. Average number of counterions within a distance $2.5 \sigma$ of another counterion scaled by the same number for an uncharged system for various packing fractions $\eta$. The chain length is $N=10$.

$>l_{B}^{*}$ increases with $l_{B}$. Thus, it is reasonable to define $l_{B}^{*}$ as the critical Bjerrum length above which counterions condense on a polyelectrolyte chain.

From Fig. 3 we see that the most probable distance between condensed counterions is $2 \sigma$. Accordingly, it is reasonable to count the number of ions within a distance $\hat{r}$ $=2.5 \sigma$ of each other. The choice guarantees that all condensed ions are taken into account. Figure 7 displays the Bjerrum length dependence of the average number of counterions $N_{\mathrm{cc}}$ within a distance of $2.5 \sigma$ of another counterion, scaled by the number of ions of an uncharged system, for various packing fractions. The figure exhibits the expected behavior: The number of ions slowly decreases for small $l_{B}$ with increasing $l_{B}$, but for $l_{B}$ above a particular Bjerrum length this number increases very rapidly, indicating counterion condensation. The critical Bjerrum length $l_{B}^{*}$ corresponds to the Bjerrum length at the minimum of $N_{\mathrm{cc}}$. In addition, we calculated $N_{\mathrm{cc}}$ within various larger distances. We find a slight dependence of $l_{B}^{*}$ on the cutoff radius $\hat{r}$. More precisely, $l_{B}^{*}$ increases with increasing $\hat{r}$. Figure 8 displays $l_{B}^{*}$ as a function of the packing fraction and for various

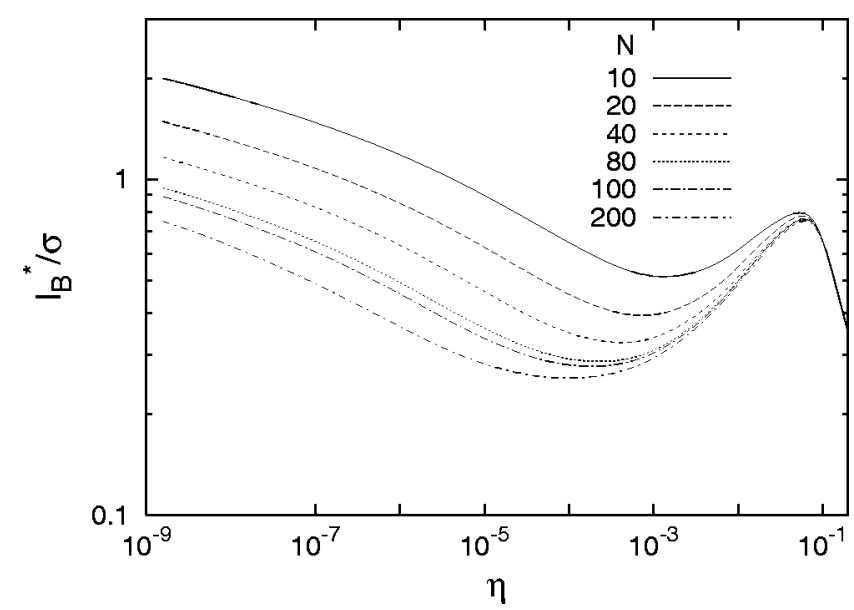

FIG. 8. Critical Bjerrum length $l_{B}^{*}$ required for counterion condensation defined in Sec. V as a function of the packing fraction and for various chain lengths.

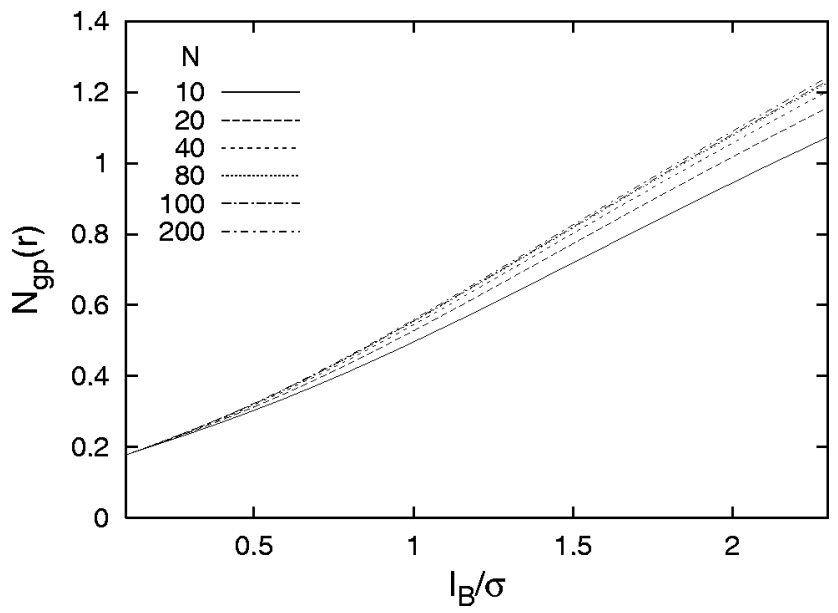

FIG. 9. Bjerrum lengths dependence of the number of condensed counterions per monomer for various chain lengths. The density is $\eta=10^{-2}$.

chain lengths. As is obvious from the figure, $l_{B}^{*}$ decreases with the packing fraction for low to moderate $\eta$ values (about $10^{-4}-10^{-3}$ depending on the chain length) and increases with $\eta$ for high densities. The drop in $l_{B}^{*}$ for $\eta$ $>0.1$ marks the breakdown of our definition for $l_{B}^{*}$ because the condensation in this density regime is dominated by packing aspects rather than the Bjerrum length. The Bjerrum length $l_{B}^{*}$ in dilute solutions is always lower for longer chains. This is in agreement with the discussion in Sec. III: The counterions condense faster on longer chains than on shorter ones. In dilute solutions, where the counterions are on the average far away from the chain, the counterions do not see the local charges on the monomers but an effective particle with a charge of $N Z_{p} e$. Therefore, the attraction between a counterion and the polymer chains is stronger for longer chains. On the other hand for high densities, $l_{B}^{*}$ is almost equal for all chain lengths. At high densities, the counterions are on the average close to a chain and the effective charge is no longer given by $N Z_{p} e$ but by a few monomer charges and therefore independent of the chain length.

Furthermore, Fig. 8 shows that the critical Bjerrum length $l_{B}^{*}$ can be quite different from $l_{B} / \sigma=1$ predicted by Manning as a condensation threshold. ${ }^{62-64}$ It should be noted that Manning's calculations were done for a hard core system in the limit of zero concentration and infinite polymer length. Figure 8 actually indicates that this value is reached with our definition of $l_{B}^{*}$ in the limit of very long chains and very dilute solutions.

Finally, we calculated the number of counterions condensed on the chain. We consider a counterion as condensed if it is within a distance of $1.5 \sigma$ of a monomer. Figure 9 shows the number $N_{\mathrm{mc}}$ of condensed ions per monomer as a function of the Bjerrum length for the density $\eta=10^{-2}$.

$N_{\text {mc }}$ increases monotonous with the Bjerrum length and is, as expected from the discussions of the previous sections, always larger for longer chains. Furthermore, we can see from Fig. 9 that the influence of the chain length is stronger for shorter chains. This indicates that $N_{\text {mc }}$ should become independent of the chain length for sufficiently long chains. This behavior is in agreement with the results of computer 
simulations. ${ }^{32}$ It should be noted that the limiting number for $N_{\text {mc }}$ at large Bjerrum lengths is two because in the complete condensed state there are two counterions within a distance of $1.5 \sigma$ from each monomer.

In Refs. 48 and 49 a different criterion for counterion condensation is provided. Investigating the counterion distribution of an infinite rod within the cell model by the Poisson-Boltzmann theory and computer simulations the authors suggest using the inflection point of the probability distribution of counterions transverse to the rod axis to separate condensed from noncondensed ions. The idea is intriguing but the criterion is difficult to apply in our threedimensional system of many chains. The inflection point criterion is useful when a counterion can uniquely be assigned to a particular chain. That is possible in computer simulations of (effectively) single chains like in the cell model. For a system of many chains the assignment of an ion to a particular chain is a difficult task ${ }^{44}$ and it is not obvious whether the inflection point criterion is useful.

There are alternative criteria to separate condensed from noncondensed ions using the pair correlation functions provided by the PRISM theory. A detailed discussion will be presented in an upcoming publication.

\section{CONCLUSION}

We have analyzed the structural properties of polyelectrolyte solutions with explicit incorporation of counterions using the PRISM integral equation theory. Particular attention has been paid to counterion condensation. The polyelectrolyte chains are modeled as rigid rods interacting with each other and the counterions through hard core repulsion and the Coulomb potential. The Bjerrum length dependence of the structure of the system was studied by the correlation functions between the different ionic species. We found that beyond a critical Bjerrum length the counterions condense on the polymer chains. We introduced a new criterium for the minimum Bjerrum length $l_{B}^{*}$ required for condensation which is, in the appropriate limit, equivalent to the Manning criterium.

It seems obvious to us that the PRISM integral equations capture the main features required to describe polyelectrolyte solutions. The next step should be the study of the charge induced conformational changes of flexible and semiflexible chains using a self-consistent approach. Investigations along that line are underway.

\section{ACKNOWLEDGMENT}

The authors gratefully acknowledge financial support by the Deutsche Forschungsgemeinschaft within the Schwerpunktsprogramm 1009.

\footnotetext{
${ }^{1}$ M. Mandel, Polyelectrolytes (Ridel, Dordrecht, 1988).

${ }^{2}$ M. Hara, Polyelectrolytes: Science and Technology (Marcel Dekker, New York, 1993).

${ }^{3}$ C. F. Foster and M. Schmidt, Adv. Polym. Sci. 120, 50 (1995).

${ }^{4}$ C. Tanford, Physical Chemistry of Macromolecules (Wiley, New York, 1961).

${ }^{5}$ Encyclopedia of Polymer Science and Engineering, edited by H. F. Mark et al. (Wiley, New York, 1990).

${ }^{6}$ F. Buchholz, Trends Polym. 99, 277 (1992).
}

${ }^{7}$ S. Förster and M. Schmid, Adv. Polym. Sci. 120, 51 (1995).

${ }^{8}$ J.-L. Barrat and J.-F. Joanny, Adv. Chem. Phys. XCIV, 1 (1996).

${ }^{9}$ Macro-ion Characterization: From Dilute Solutions to Complex Fluids, edited by K. Schmitz (American Chemical Society, Washington, DC, 1994).

${ }^{10}$ J. Hayter, G. Janninck, F. Brochard-Wyart, and P. de Gennes, J. Phys. (France) Lett. 41, 451 (1980).

${ }^{11}$ L. Wang and V. Bloomfield, Macromolecules 23, 804 (1990).

${ }^{12}$ T. Witten and P. Pincus, Europhys. Lett. 3, 315 (1987).

${ }^{13}$ J. Bodycomb and M. Hara, Macromolecules, 27, 7369 (1994).

${ }^{14}$ M. Nierlich et al., J. Phys. (Paris) 40, 701 (1979).

${ }^{15}$ M. Nierlich, F. Boue, A. Lapp, and R. Oberthür, Colloid Polym. Sci. 263, 955 (1985).

${ }^{16}$ G. Jannink, Makromol. Chem., Macromol. Symp. 1, 67 (1986).

${ }^{17}$ M. Sedlak and E. Amis, J. Chem. Phys. 96, 817 (1992).

${ }^{18}$ S. Förster, M. Schmidt, and M. Antonietti, J. Phys. Chem. 96, 4008 (1992)

${ }^{19}$ P. G. de Gennes, Scaling Concepts in Polymer Physics (Cornell University Press, Ithaca, NY, 1979)

${ }^{20}$ J. des Cloiseaux and G. Jannink, Les Polymeres en Solution, Leur Modelisation et Leur Strucure (Editions de Physique, Paris, 1985).

${ }^{21}$ M. Doi and S. F. Edwards, The Theory of Polymer Dynamics (Oxford University Press, Oxford, 1986).

${ }^{22}$ T. Odijk, J. Polym. Sci. 15, 477 (1977).

${ }^{23}$ J. Skolnick and M. Fixman, Macromolecules 10, 944 (1977).

${ }^{24}$ T. Odijk and A. C. Houwaart, J. Polym. Sci. 16, 627 (1978).

${ }^{25}$ D. Adams and G. Dubey, J. Comput. Phys. 72, 156 (1987).

${ }^{26}$ B. Smit, Computer Simulations in Chemical Physics (Kluwer Academic, Dordrecht, 1993).

${ }^{27}$ G. A. Christos and S. L. Carnie, J. Chem. Phys. 91, 439 (1989).

${ }^{28}$ J. L. Barrat and D. Boyer, J. Phys. II 3, 343 (1993).

${ }^{29}$ M. Stevens and K. Kremer, Macromolecules 26, 4717 (1993).

${ }^{30}$ M. Stevens and K. Kremer, Chem. Phys. Lett. 71, 2228 (1993).

${ }^{31}$ M. Stevens and K. Kremer, J. Chem. Phys. 103, 1669 (1995).

${ }^{32}$ R. G. Winkler, M. Gold, and P. Reineker, Phys. Rev. Lett. 80, 3731 (1998).

${ }^{33}$ K. S. Schweizer and J. G. Curro, Phys. Rev. Lett. 58, 246 (1987).

${ }^{34}$ K. S. Schweizer and J. G. Curro, Macromolecules 20, 1928 (1987).

${ }^{35}$ K. S. Schweizer and J. G. Curro, J. Chem. Phys. 87, 1842 (1987).

${ }^{36}$ K. S. Schweizer and J. G. Curro, Adv. Polym. Sci. 116, 319 (1995).

${ }^{37}$ K. S. Schweizer and A. Yethiraj, J. Chem. Phys. 98, 9053 (1993).

${ }^{38}$ K. S. Schweizer and J. G. Curro, J. Chem. Phys. 89, 3342 (1982).

${ }^{39}$ K. G. Honnell, K. S. Schweizer, and J. G. Curro, Macromolecules 23 , 3496 (1990).

${ }^{40}$ D. A. McQuarrie, Statistical Mechanics (Harper and Row, New York, 1979).

${ }^{41}$ M. Dymitrwska and L. Belloni, J. Chem. Phys. 109, 4659 (1998).

${ }^{42}$ C. Y. Shew and A. Yethiraj, J. Chem. Phys. 106, 5706 (1997).

${ }^{43}$ L. Belloni, Chem. Phys. 99, 43 (1985).

${ }^{44}$ M. Dymitrwska and L. Belloni, J. Chem. Phys. 111, 6633 (1999).

${ }^{45}$ C. Y. Shew and A. Yethiraj, J. Chem. Phys. 109, 5162 (1998).

${ }^{46}$ C. Y. Shew and A. Yethiraj, J. Chem. Phys. 110, 11599 (1999).

${ }^{47}$ L. Harnau and P. Reineker, J. Chem. Phys. 112, 437 (2000).

${ }^{48}$ M. Deserno, C. Holm, and S. May, Macromolecules 33, 199 (2000)

${ }^{49}$ L. Belloni, M. Drifford, and P. Turq, Chem. Phys. 83, 147 (1984).

${ }^{50}$ J. P. Hansen and I. R. McDonald, Theory of Simple Liquids (Academic, London, 1986).

${ }^{51}$ D. Chandler and H. C. Andersen, J. Chem. Phys. 57, 1930 (1972).

${ }^{52}$ K. S. Schweizer and J. G. Curro, Macromolecules 21, 3070 (1988).

${ }^{53}$ A. Yethiraj and C. K. Hall, J. Chem. Phys. 93, 4453 (1990).

${ }^{54}$ A. Yethiraj and C. K. Hall, J. Chem. Phys. 96, 797 (1992).

${ }^{55}$ D. Laria, D. Wu, and D. Chandler, J. Chem. Phys. 95, 4444 (1991).

${ }^{56}$ E. E. Maier, S. F. Schulz, and R. Weber, Macromolecules 21, 1544 (1988).

${ }^{57}$ S. F. Schulz, E. E. Maier, and R. Weber, J. Chem. Phys. 90, 7 (1989).

${ }^{58}$ E. E. Maier, R. Krause, M. Deggelmann, M. Hagenbüchle, R. Weber, and S. Fraden, Macromolecules 25, 1125 (1992).

${ }^{59} \mathrm{~T}$. Odijk, Macromolecules 12, 688 (1979).

${ }^{60}$ J. G. Jensen, R. J. Mashl, R. F. Bruinsma, and W. M. Gelbart, Phys. Rev. Lett. 78, 2477 (1997)

${ }^{61}$ A. Hanke, E. Eisenriegler, and S. Dietrich, Phys. Rev. E 59, 6853 (1999).

${ }^{62}$ G. S. Manning, J. Chem. Phys. 51, 924 (1969).

${ }^{63}$ G. S. Manning, J. Chem. Phys. 51, 934 (1969).

${ }^{64}$ G. S. Manning, J. Chem. Phys. 51, 3249 (1969). 